\title{
Rendezvous for Heterogeneous Spectrum-Agile Devices
}

\author{
Shan-Hung Wu, Ching-Chan Wu, Wing-Kai Hon, Kang G. Shin \\ \{shwu,wkhon\}@cs.nthu.edu.tw, ccwu@netdb.cs.nthu.edu.tw, kgshin@eecs.umich.edu
}

\begin{abstract}
Cognitive radio (CR) is intended to meet the exponentially growing demand for spectrum by allowing for opportunistic utilization of idle legacy channels. Rendezvous, where two radios complete handshaking in an idle channel, is a key step for "stranger" (unknown to each other) CRs to start communication. However, none of existing algorithms guarantee rendezvous for heterogeneous or stranger CRs with different spectrum-sensing capabilities, in spite of the fact that (i) a wide variety of mobile devices are equipped with heterogeneous radios and (ii) there are numerous applications requiring efficient rendezvous for heterogeneous radios/CRs.

In this paper, we propose a new channel hopping algorithm, called Heterogeneous Hopping $(\mathrm{HH})$, that guarantees rendezvous without assuming existence of a universal channel set that can be sensed by all radios. HH is realized with a two-layer design that harmonizes the fixed-short-cycle and parity-alignment techniques we propose here, in order to guide CRs to rendezvous in two complementary situations resulting from the different capabilities of mobile wireless devices. To best of our knowledge, $\mathrm{HH}$ is the first channel-hopping scheme that guarantees rendezvous between heterogeneous radios. Our in-depth evaluation has shown $\mathrm{HH}$ to be significantly faster than simple extensions of existing schemes. Moreover, the latter cannot guarantee successful rendezvous, either.
\end{abstract}

\section{INTRODUCTION}

In recent years, we have been witnessing an exponential growth of mobile wireless devices and applications around the globe, generating a huge demand for the radio spectrum. Spectrum resources, such as channels, grow at a much slower pace and the current static spectrum allocation is expected to be unable to meet this demand. Measurement studies [1], [15] show that the allocated radio spectrum is severely underutilized by primary users (PUs) in time and space, thus leaving many spectrum holes or idle channels. To utilize these idle channels, secondary users (SUs), or simply radios/nodes, dynamically tune to different idle channels to communicate with one another.

Conventionally, an ad hoc cognitive radio network (CRN) is designed to support communications among a small group of users who are acquaintances/friends to each other. However, various wireless devices have recently been made spectrumagile, allowing a large-scale ad hoc CRN to be formed between "stranger" (i.e., unknown to each other) SUs [11]. This CRN formed with stranger devices is useful or necessary for many applications and is likely to contain heterogeneous devices with different sensing capabilities. For example, in a disasterstricken area, people should be able to opportunistically use any wireless devices available to share and send/receive emergency messages. For data collection/dissemination in mobile sensor networks (for, say, wildlife conservation, traffic monitoring, or battlefield communications), sensors (as SUs)

978-1-4799-3360-0/14/\$31.00 (c)2014 IEEE

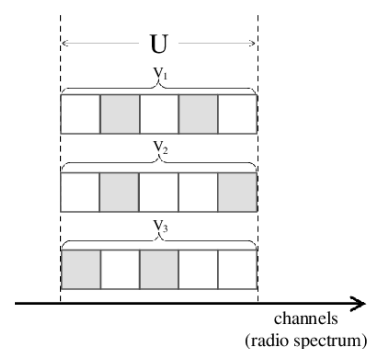

(a)

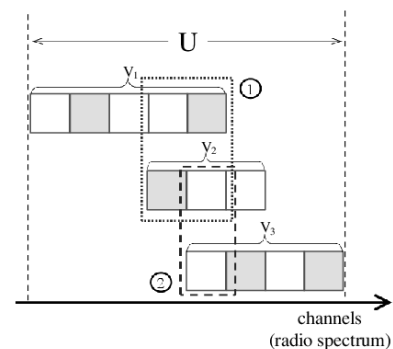

(b)
Figure 1. Channel-availability models. Channels occupied by PUs are shaded: (a) the asymmetric model, where $V_{i}=V_{j}, \forall i, j$; (b) the heterogeneous model, which allows $V_{i} \neq V_{j}$ for some $i$ and $j$.

can continue to collect/transmit critical data without getting affected by the interference in certain areas. For the Internet of Things (IoT), "things," as SUs, should be able to access the status of other things directly without centralized coordination between different types of things.

Rendezvous is the first key step for "stranger" CRs to be able to communicate with each other. By rendezvous, we mean that two in-range radios complete handshaking in an idle channel for neighbor discovery and/or data communications. One common way to guide radios to rendezvous is to use Channel Hopping (CH) [7], [13], [14], [17], [20], [24], [25]. A channel-hopping scheme, programmed in every radio, evenly divides every radio's time into time slots and requires the radio to hop to some channel at each time slot. The sequence of channels that the radio hops to in consecutive slots is called the channel-hopping sequence. It is the $\mathrm{CH}$ scheme that generates the $\mathrm{CH}$ sequences for different radios and guarantees rendezvous by guiding any two neighboring radios to hop to the same channel at two overlapping slots within a finite delay called the time to rendezvous (TTR). In contrast to other techniques such as the Common Control Channel (CCC) [8], [9], [12], [18], [19], [22], the $\mathrm{CH}$ scheme allows radios to determine and follow their own $\mathrm{CH}$ sequences in a distributed manner, thereby withstanding single-point-of-failures. Also, different radio pairs can rendezvous in different channels, thus avoiding network congestion. In addition, some $\mathrm{CH}$ schemes [4], [5], [7], [14], [20], [27] can guarantee rendezvous even when timers are not synchronized across radios.

However, existing $\mathrm{CH}$ hopping schemes are not designed for a large network of stranger CR nodes. Let $V_{i}$ be the set of channels that radio $i$ is capable of sensing with, and $P_{i} \subseteq V_{i}$ be a set of channels in $V_{i}$ that are found occupied by PUs. 
Most existing $\mathrm{CH}$ schemes assume an asymmetric model, ${ }^{1}$ as shown in Fig. 1(a), where all devices have the same spectrumsensing capability, i.e., $V_{i}=V_{j}, \forall i \neq j$. Although this model is reasonable for a small group of acquaintances/friends, we argue that a heterogeneous model (Fig. 1(b)), where $V_{i} \neq V_{j}$ for some $i$ and $j$, is more realistic for rendezvous between stranger CRs due to the inherent heterogeneity of wireless devices. Note that the size of the universal channel set $U=$ $\bigcup_{i} V_{i}$ in this model can be much larger than $\left|V_{i}\right|$ of radio $i$.

In this paper, we investigate rendezvous between stranger CRs with the heterogeneous model. Our goal is to guarantee rendezvous between any two neighboring radios $i$ and $j$ as long as $\left(V_{i} \backslash P_{i}\right) \cap\left(V_{j} \backslash P_{j}\right) \neq \emptyset .^{2}$ We initially tried to extend existing $\mathrm{CH}$ schemes but found that such an extension cannot guarantee rendezvous between all pairs of neighboring radios, and even if it guarantees the rendezvous for a given pair, the maximum TTR (MTTR) will be unacceptably long (at least $O\left(|U|^{2}\right)$, measured in number of slots, usually taking minutes or hours). Rendezvous between stranger CRs is thus an important, unsolved problem.

We identify two new requirements that must be met by the $\mathrm{CH}$ schemes for stranger CRs, and design from scratch a new $\mathrm{CH}$ scheme, called Heterogeneous Hopping $(\mathrm{HH})$, to meet these requirements. $\mathrm{HH}$ has the following salient features.

- It guarantees rendezvous between two neighboring radios $i$ and $j$ as long as $\left(V_{i} \backslash P_{i}\right) \cap\left(V_{j} \backslash P_{j}\right) \neq \emptyset$. Note that there are other schemes [23] that assume the heterogeneous model. However, like our naive extensions (to be discussed), they cannot guarantee all-pair rendezvous (a counter example is given in Section VI). To best of our knowledge, $\mathrm{HH}$ is the first $\mathrm{CH}$ scheme that guarantees rendezvous between stranger CRs.

- As the timer synchronization between radios is usually hard to achieve (especially before rendezvous), HH takes into account the clock skews between radios and supports both synchronous and asynchronous environments.

- The MTTR given by HH is bounded by $O\left(\left|V_{i}\right|\left|V_{j}\right|\right)$ in number of slots and is independent of $|U|$. Note that the heterogeneous model is a generalization of the asymmetric model. Therefore, $\mathrm{HH}$ is applicable to asymmetric environments where $V_{i}=V_{j}=U$. In this case, $\mathrm{HH}$ produces $O\left(|U|^{2}\right)$ MTTR, which is as fast as the best known CH schemes [14], [20], [27] for asynchronous environments.

Our extensive simulation results show that $\mathrm{HH}$ is $10 \times$ faster than extensions of existing schemes in terms of average TTR (let alone their inability of guaranteeing a successful rendezvous).

The rest of this paper is organized as follows. Section II discusses the limitations of existing $\mathrm{CH}$ schemes and identifies important missing requirements. Section III describes principles behind $\mathrm{HH}$ while Section IV details the design of $\mathrm{HH}$ and verifies its ability of guaranteeing rendezvous. Section V evaluates the performance of $\mathrm{HH}$ and Section VI discusses related work. Finally, Section VII concludes the paper.

\footnotetext{
${ }^{1}$ The asymmetric model is named against the symmetric model, where $V_{i}=$ $V_{j}$ and $P_{i}=P_{j}$, assumed by early $\mathrm{CH}$ schemes. The authors of [10], [15] reported that the PU-occupancy changes over time and across geographic space, making the asymmetric model more acceptable.

${ }^{2}$ There is no way for radios $i$ and $j$ to rendezvous with each other if $\left(V_{i} \backslash P_{i}\right) \cap\left(V_{j} \backslash P_{j}\right)=\emptyset$.
}

Table I

NOTATION.

\begin{tabular}{r|l}
\hline Variable & \multicolumn{1}{c}{ Description } \\
\hline \hline$U$ & The set of universal channels. \\
$V_{i}$ & The set of channels sensible by radio $i$. \\
$P_{i}$ & The set of PU occupied channels detected \\
& by radio $i$. \\
start $_{i}$ & The starting channel of $V_{i}$. \\
$t_{i}^{[z]}$ & The $z^{t h}$ time slot of radio $i$. \\
$S_{i}^{[x]}$ & CH sequence of radio $i$ in the $x^{t h}$ \\
$s_{i}^{[x, y]}$ & cycle/round. \\
$F_{i}$ & The $y^{t h}$ element in $S_{i}^{[x]}$. \\
$R_{i}$ & The fixed subsequence of $S_{i}$. \\
$N_{i}$ & The parity subsequence of $S_{i}$. \\
$k_{i}$ & Amount of rotation of $R_{i}$.
\end{tabular}

\section{Limitations of Existing Schemes And REMAining CHALlENGES}

In this Section, we discuss the limitations of existing $\mathrm{CH}$ schemes when applied to heterogeneous CRNs, and then identify the requirements that must be met for new $\mathrm{CH}$ schemes. Table I summarizes the notation to be used throughout the paper.

Suppose the universal spectrum can be divided into a set $U=\{0,1, \cdots,|U|-1\}$ of channels. Each radio $i$ is capable of sensing a range of spectrum consisting of a set $V_{i} \subseteq U$ of contiguous channels [1], [2], [10], [15], [16]. Each channel in the capability set $V_{i}$ is either occupied by nearby PUs or available for opportunistic usage by SUs, and we let $P_{i} \subseteq V_{i}$ be the set of PU-occupied channels that radio $i$ detects. The symmetric model assumes $V_{i}=V_{i}=U$ and $P_{i}=P_{j}, \forall i \neq j$, while the asymmetric model allows $P_{i} \neq P_{j}$ for some $i$ and $j$. The heterogeneous model further allows $V_{i} \neq V_{j}$ for some $i$ and $j$.

A $\mathrm{CH}$ scheme is usually implemented at the MAC layer. A radio $i$ obtains its own $\mathrm{CH}$ sequence $S_{i}=\left[s_{i}^{[0]}, s_{i}^{[1]}, \cdots\right]$, where $s_{i}^{[z]} \in V_{i}$, from a $\mathrm{CH}$ scheme, and hops to channel $s_{i}^{[0]}$ at slot $t_{i}^{[0]}, s_{i}^{[1]}$ at slot $t_{i}^{[1]}$, and so on, as shown in Fig. 2. A $\mathrm{CH}$ sequence $S_{i}$ can be partitioned evenly into cycles $S_{i}^{[x]}=\left[s_{i}^{[x, 0]}, s_{i}^{[x, 1]}, \cdots, s_{i}^{\left[x,\left|S_{i}^{[x]}\right|\right]}\right]$, where $s_{i}^{[x, y]}$ denotes the $y^{t h}$ element in the $x^{t h}$ cycle. Note that $s_{i}^{[z]}=s_{i}^{\left[x \cdot\left|S_{i}^{[x]}\right|+y\right]}$. Different cycles $S_{i}^{[x]}$ and $S_{i}^{\left[x^{\prime}\right]}$ must have the same cycle length $\left|S_{i}^{[x]}\right|=\left|S_{i}^{\left[x^{\prime}\right]}\right|=T$, but can be assigned different channels in the same position $y$ (i.e., $s_{i}^{[x, y]} \neq s_{i}^{\left[x^{\prime}, y\right]}$ ).

To evaluate the performance of a $\mathrm{CH}$ scheme, one common metric is the Maximum Time to Rendezvous (MTTR), which measures the maximum time (in number of slots) required for two radios to rendezvous. The shorter the MTTR the better.

The most naive way to generate a $\mathrm{CH}$ sequence for radio $i$ is to randomly assign a channel from $V_{i}$ to each time slot. A study, called Modified Modular Clock (MMC) [25], shows that radios rendezvous with a high probability by following this randomized strategy. However, randomized schemes cannot guarantee rendezvous within finite time, so they cannot support QoS at the network or application layers (e.g., to choose a route satisfying a delay constraint). Recent efforts [3], [4], 


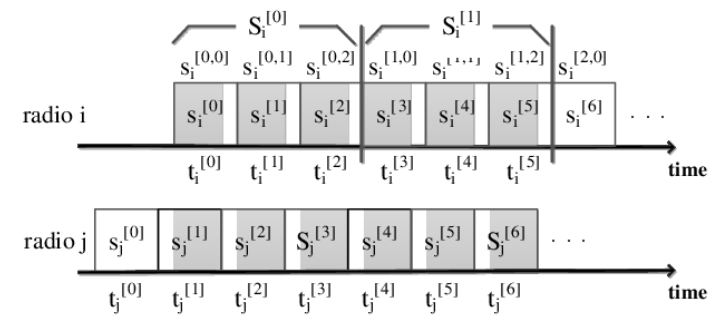

Figure 2. Time slots and $\mathrm{CH}$ sequences. The slot $t_{i}^{[1]}$ is time-overlapping with $t_{j}^{[2]}$, but not with $t_{j}^{[1]}$ and $t_{j}^{[3]}$. In terms of time-overlapping, we only need to consider the time skews that are integer multiples of a time slot.

(a)

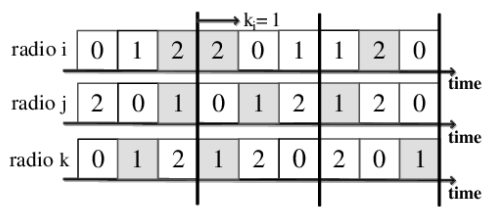

(b)

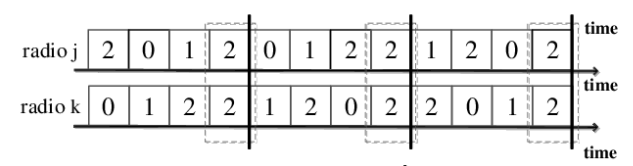

(c)

\begin{tabular}{|c|c|c|c|c|c|c|c|c|c|c|c|c|c|c|c|}
\hline radio $j$ & 2 & 0 & & 1 & 2 & 2 & 2 & 2 & 0 & 1 & 2 & 2 & 2 & 2 & 2 \\
\hline radio $\mathrm{k}$ & 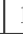 & 2 & & 2 & 2 & 2 & 2 & 1 & 2 & 0 & 2 & 2 & 2 & 2 & \\
\hline
\end{tabular}

Figure 3. Techniques for generating $\mathrm{CH}$ sequences. Let $V=U=\{0,1,2\}$ : (a) Prime-and-rotating, where $T=|U|=3, k_{i}=1, k_{j}=k_{k}=2$, $P_{i}=\{2\}$, and $P_{j}=P_{k}=\{1\} ;$ (b) Rotating-and-parity, where $k_{j}=k_{k}=1$ (parity slots are marked in the boxes; (c) Asynchronous rotating-and-parity, where $T=7$ (it takes 4 slots-larger than $T / 2$ - to form a majority quorum).

[20], [25], [27] have been made to guarantee a bounded MTTR under the asymmetric model where $V_{i}=U$.

\section{A. Techniques for Guaranteeing Rendezvous}

To generate the $\mathrm{CH}$ sequences that guarantee rendezvous when $V_{i}=U$, a common technique employed by existing $\mathrm{CH}$ schemes, which we call prime-and-rotating, is to partition $S_{i}$ into cycles $S_{i}^{[x]}$ of length $T$ being the least prime number larger than or equal to $|U|$, and define $S_{i}^{[x]}$ according to the following rule:

$$
s_{i}^{[x, y]}= \begin{cases}v_{i}^{(y)}, & x=0 \text { and } y<|U|, \\ \text { an arbitrary channel in } U, & x=0 \text { and } y \geq|U|, \\ s_{i}^{\left[x-1,\left(y-k_{i}\right) \bmod T\right],} & \text { otherwise, }\end{cases}
$$

where $v_{i}^{(y)}$ is the $y^{t h}$ smallest element in $U$ (indexed from 0 ) and $k_{i}, 0 \leq k_{i} \leq|U|-1$, is an arbitrary integer chosen by radio $i$. Basically, the first cycle $S_{i}^{[0]}$ contains the "augmented" $U$ whose size is a prime number, and then in each cycle $S_{i}^{[x-1]}$, channels are rotated $k_{i}$ slots forward to produce the next cycle $S_{i}^{[x]}$. Fig. 3(a) shows an example of this. It can be easily shown [20] that with this scheme, a pair of radios $i$ and $j$ can rendezvous within $O\left(|U|^{2}\right)$ slots (i.e., MTTR) if they chose different amounts of rotation $\left(k_{i} \neq k_{j}\right)$. In Fig. 3(a), radio $i$ will eventually rendezvous with radio $j$ in channel 0 . However, two radios cannot rendezvous if they happen to choose the same amount of rotation, as shown by radios $j$ and $k$ in Fig. 3(a). To solve this problem, another technique, called rotating-and-parity, is commonly used by the involved radios together. This technique requires radio $i$ picking the amount of rotation $k_{i}$ to hop to the channel $k_{i}$ at some predefined parity slots in each cycle, as shown in Fig. 3(b). In this case, $T$ is equals to the prime plus the number of parity slots in each cycle. The trick is that if two radios accidentally pick the same amount of rotation, then they will rendezvous at the parity slots.

\section{B. Techniques for Coping with Asynchronous Timers}

The above examples implicitly assume that the timers of radios are synchronized. In an asynchronous environment, there is no guarantee of rendezvous between radios $j$ and $k$ in Fig. 3(b) since the parity slots may not be aligned with each other. To guarantee rendezvous, we need to ensure the overlap of parity slots in the presence of time skews. Note that in spite of asynchronous timers, each slot of a radio must overlap with exactly one slot of another radio during an interval (shaded in Fig. 2) longer than a half slot.

Definition 1 (Time-overlap). Two slots $t_{i}^{[z]}$ and $t_{j}^{\left[z^{\prime}\right]}$ of radios $i$ and $j$, respectively, are said to time-overlap if they overlap for an interval longer than a half slot.

For example, in Fig. 2, the slot $t_{i}^{[1]}$ time-overlaps with $t_{j}^{[2]}$, but not with $t_{j}^{[3]}$. Suppose the duration of each slot is so configured that two radios in a channel can handshake within a half slot, then rendezvous in an asynchronous environment can be defined as follows.

Definition 2 (Rendezvous). Two radios $i$ and $j$ are said to rendezvous if they hop to some common available channel $c$ at a pair of time-overlapping slots, i.e., $s_{i}^{[z]}=s_{j}^{\left[z^{\prime}\right]}=c$ for some $z, z^{\prime}$, and $c$, where $t_{i}^{[z]}$ time-overlaps with $t_{j}^{\left[z^{\prime}\right]}$ and $c \in\left(V_{i} \backslash P_{i}\right) \cap\left(V_{j} \backslash P_{j}\right)$.

The above definitions simplify the design of $\mathrm{CH}$ schemes for asynchronous $\mathrm{CRNs}$, as we only need to consider the time skews that are equal to integer multiples of a time slot. That is, we can safely assume that slot boundaries are always aligned across radios. With this simplification, the concept of cyclic quorums [21] can be adopted to ensure a time-overlap between the parity slots in each cycle [4]-[6], [23]. For example, as shown in Fig. 3(c), each radio can hop to the parity channel $k_{i}$ at parity slots specified by a majority quorum (i.e., a set of slots with size greater than $T / 2$ ). Note that despite the time skew, one of the parity slots of radio $j$ in each cycle must time-overlap with a parity slot of $k$, thereby guaranteeing rendezvous.

\section{Limitations and Requirements when $V_{i} \neq V_{j}$}

Radios in CRNs with the heterogeneous model may have different spectrum sensing capabilities, i.e., $V_{i} \neq V_{j}$ for some $i$ and $j$. To apply existing asymmetric $\mathrm{CH}$ schemes (which require $V_{i}=V_{j}=U$ to ensure cycle lengths to be the same) to heterogeneous CRNs, we can assume that radios have the same nominal capability set $V$ containing the full spectrum (i.e., $V=U$ ), and regard those channels out of the true capability set $V_{i}$ of radio $i$ as PU-occupied all time (i.e., $P_{i}$ contains $V \backslash V_{i}$ ). However, this naive extension suffers from two drawbacks. First, the parity channel $k_{i}$ used in the rotatingand-parity technique (see Figs. 3(b)) may not be sensible by a radio device (i.e., $k_{i} \in V$ but $k_{i} \notin V_{i}$ ), and we lose the 


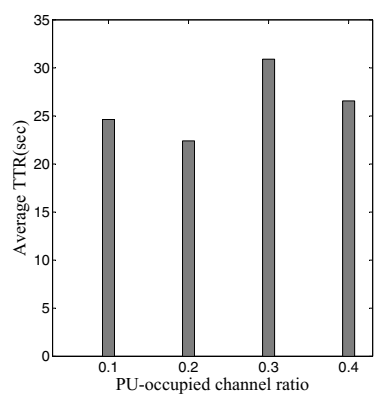

(a)

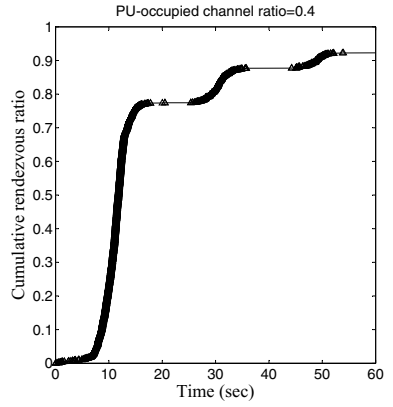

(b)
Figure 4. Problems of naive extension by creating nominal $V=U$ : (a) average TTR vs. ratio of PU-occupied channels to $U$; (b) cumulative rendezvous ratio (to the set $D$ of all radios) vs. simulation time given that the PU-occupied ratio is set to 0.4 .

rendezvous guarantee for radios picking the same amount of rotation. Second, due to the variety of wireless devices, the size of $U$ in heterogeneous CRNs can be very large. For example, if we consider all devices operating in the spectrum ranging from $30 \mathrm{MHz}$ to $3 \mathrm{GHz}$ and assume $5 \mathrm{MHz}$ channel width, there are about 600 channels in $U$ [15]. The $O\left(|U|^{2}\right)$ slots MTTR given by the prime-and-rotating technique (shown in Fig. 3(a)) implies that two radios need at most 10 hours to rendezvous with each other provided $100 \mathrm{~ms}$ time slots.

To see how the naive extension performs in practice, we simulate a CRN with the heterogeneous model, where there are five types of radios. Different types of radios have different but overlapping spectrum sensing capabilities. There are three radios for each type. More details are provided in Section V. Fig. 4(a) shows that on average, radios need to wait for more than 20 seconds to start communications. Fig. 4(b) shows that nearly $10 \%$ of the radios cannot start communications even after 60 seconds. This may be due to either the long MTTR, or lack of rendezvous guarantee of the rotating-andparity technique.

Based on the above observations, we identify two requirements for a $\mathrm{CH}$ scheme to be useful in heterogeneous CRNs:

1) The MTTR given by the $\mathrm{CH}$ scheme should be independent of $|U|$ to avoid excessive delays.

2) Since there is no way to prevent radios from making the same decision accidentally (e.g., picking the same amount of rotation in the prime-and-rotating technique), a "parity" technique like rotating-and-parity is inevitable. But this technique must ensure that the parity channel belongs to $V_{i} \backslash P_{i}$.

\section{Principles of Heterogeneous Hopping}

We now present the principles behind the proposed Heterogeneous Hopping $(\mathrm{HH})$ scheme that guarantees rendezvous between stranger SUs under the heterogeneous model.

Definition 3 (Capability/availability-overlap). Two radios $i$ and $j$ are said to have capability-overlap if they can both sense at least one common channel, i.e., $V_{i} \cap V_{j} \neq \emptyset$, and have availability-overlap if $\left(V_{i} \backslash P_{i}\right) \cap\left(V_{j} \backslash P_{j}\right) \neq \emptyset$.

Dotted-line boxes (1) and (2) in Fig. 1(b) show the capabilityand availability-overlap examples, respectively. Note that two radios cannot rendezvous if no availability-overlap exists between them. Our problem is formally defined as:

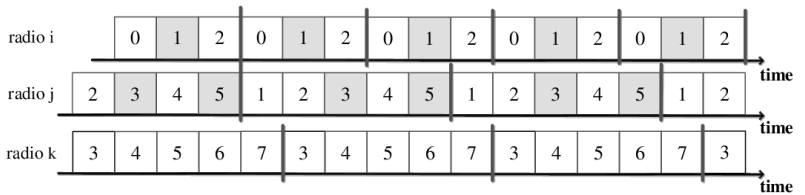

Figure 5. The fixed-short-cycle technique, where $V_{i}=\{0,1,2\}, P_{i}=\{1\}$, $V_{j}=\{1,2,3,4,5\}, P_{j}=\{3,5\}, V_{k}=\{3,4,5,6,7\}$, and $P_{k}=\emptyset$.

Problem 4. Given a pair of capability-overlapping radios $i$ and $j$ in a network, design a $\mathrm{CH}$ scheme that can yield two $\mathrm{CH}$ sequences $S_{i}$ and $S_{j}$, and guarantee the two radios to rendezvous within a finite time by following $S_{i}$ and $S_{j}$, respectively, if they have availability-overlap, even in the presence of a time skew between them.

We propose to solve this problem by designing $\mathrm{HH}$ to meet the requirements discussed in Section II, as described next.

\section{A. The Fixed-Short-Cycle Technique}

Channel hopping, in general, lets a radio hop to channels "blindly" without knowing the other radio's sequence, so rendezvous between the two may succeed after a finite number of blind trials/cycles. As we can see in Section II-C, the cycle lengths of $\mathrm{CH}$ sequences determine the maximum period of blind trials (i.e., MTTR). Therefore, the first requirement implies that any scheme should generate $\mathrm{CH}$ sequences whose cycle lengths are independent of $|U|$.

We adopt a fixed-short-cycle technique that reduces the cycle length of a $\mathrm{CH}$ sequence to $O\left(\left|V_{i}\right|\right)$ slots. Let $F$ denote the newly-defined $\mathrm{CH}$ sequence. For each radio $i$ with capability $V_{i}$, we first let the cycle length $\left|F_{i}^{[x]}\right|$ be the least prime larger than or equal to $\left|V_{i}\right|$, and then assign the channels to $F_{i}^{[x]}$ using the following rule:

$f_{i}^{[x, y]}= \begin{cases}v_{i}^{(y)}, & x=0 \text { and } y<\left|V_{i}\right|, \\ \text { an arbitrary element of } V_{i}, & x=0 \text { and } y \geq\left|V_{i}\right|, \\ f_{i}^{[x-1, y]}, & \text { otherwise, }\end{cases}$

where $v_{i}^{(y)}$ is the $y^{t h}$ smallest element in $V_{i}$ (indexed from 0). Fig. 5 shows an example. Basically, all cycles $F_{i}^{[x]}$ contain the "augmented" $V_{i}$ whose size is a prime number. Unlike the prime-and-rotating technique shown in Fig. 3(a), there is no rotation from cycle to cycle, and we do not rely on the rotation with the same cycle length to guide rendezvous. Instead, we use the difference between the cycle lengths to guarantee rendezvous:

Lemma 5. Let $p$ be a prime and $m, m \neq 0$, be an integer coprime with $p$. Then, for any integer $d$, the integers $d, d+$ $m, d+2 m, \cdots, d+(p-1) m$ are all distinct under modulo- $p$ arithmetic.

Proof: Suppose, on the contrary, that $d+x m \equiv d+y m$ $(\bmod p)$, for some $0 \leq x<y \leq p-1$. This implies that $(y-x) m \equiv 0 \quad(\bmod p)$, which is a contradiction since $p$ is a prime and both $y-x$ and $m$ are coprime with $p$.

Theorem 6. Given two availability-overlapping radios $i$ and $j$ that obtain the CH sequences $F_{i}$ and $F_{j}$ by following Eq. (1). The MTTR between radios $i$ and $j$ is bounded by $O\left(\left|V_{i}\right|\left|V_{j}\right|\right)$ regardless of their time skews as long as $\left|F_{i}^{[x]}\right| \neq\left|F_{j}^{[x]}\right|$. 


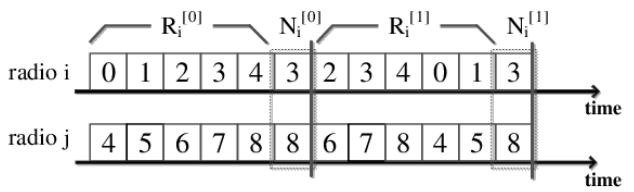

Figure 6. The modulo problem of parity-then-rotating technique. Given $V_{i}=\{0,1,2,3,4\}, n_{i}=3, V_{j}=\{4,5,6,7,8\}$, and $n_{j}=8$, the radios $i$ and $j$ have the same rotation amount $3 \equiv 8(\bmod 5)$ in $R_{i}$ and $R_{j}$, but distinct parity channels in $N_{i}$ and $N_{j}$.

Proof: Without loss of generality, suppose $f_{i}^{[x, a]}$ for some $a$ is a common available channel (i.e., $f_{i}^{[x, a]} \in\left(V_{i} \backslash P_{i}\right) \cap\left(V_{j} \backslash P_{j}\right)$ ). Let $m=\left|F_{i}^{[x]}\right|$ and $p=\left|F_{j}^{[x]}\right|$. By definition, $m$ and $p$ are coprime. If at the first cycle $f_{i}^{[0, a]}$ time-overlaps with $f_{j}^{[c, d]}$, then when radio $i$ hops to $f_{i}^{[1, a]}, f_{i}^{[2, a]}, \cdots$, and $f_{i}^{[p-1, a]}$ in the following cycles, they will have a time-overlap

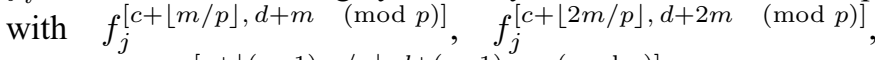
$\cdots, \quad$ and $\left.f_{j}^{[c+\lfloor(p-1) m / p\rfloor, d+(p-1) m}(\bmod p)\right]$, respectively. These $\left.f_{j}^{[c+\lfloor k m / p\rfloor, d+k m}(\bmod p)\right], 0 \leq k<p$, are all distinct under the modulo- $p$ arithmetic by Lemma 5 , so that one of them must be the same channel as $f_{i}^{[x, a]}$. The rendezvous will occur within $O\left(\left|F_{i}^{[x]}\right|\left|F_{j}^{[x]}\right|\right)=O\left(\left|V_{i}\right|\left|V_{j}\right|\right)$ slots.

\section{B. Parity-Alignment}

The $\mathrm{CH}$ sequences based on the fixed-short-cycle technique cannot guarantee rendezvous when $\left|F_{i}^{[x]}\right|=\left|F_{j}^{[x]}\right|$, as shown for radios $j$ and $k$ in Fig. 5. Note that this problem occurs only when $\left|F_{i}^{[x]}\right|=\left|F_{j}^{[x]}\right|$. To solve this problem, we need to define new, specialized $\mathrm{CH}$ sequences $G_{i}$ and $G_{j}$ that guarantee rendezvous for this case and require radios $i$ and $j$ to hop to those channels defined in $G_{i}$ and $G_{j}$ periodically at some time-overlapping slots.

Let's review again the rotating-and-parity technique shown in Fig. 3(b). Applying it to $G_{i}$, we obtain $R_{i}^{[x]}$ and $N_{i}^{[x]}$, the subsequences of $G_{i}^{[x]}$ corresponding to the rotating and parity slots in $G_{i}^{[x]}$ respectively (see Fig. 6), where $R_{i}^{[x]}$ is defined as:

$$
r_{i}^{[x, y]}=\left\{\begin{array}{ll}
f_{i}^{[x, y]}, & x=0, \\
r_{i}^{\left[x-1,\left(y-k_{i}\right) \bmod \left|R_{i}^{[x]}\right|\right],}, & \text { otherwise, }
\end{array},\right.
$$

$k_{i}$ is the amount of rotation, and $N_{i}^{[x]}$ contains the parity channel $n_{i}$ set to $k_{i}$. Note that $\left|R_{i}^{[x]}\right|=\left|F_{i}^{[x]}\right|=\left|F_{j}^{[x]}\right|=\left|R_{j}^{[x]}\right|$. The idea is to guide nodes $i$ and $j$ to rendezvous using $R_{i}^{[x]}$ and $R_{j}^{[x]}$ if $k_{i} \neq k_{j}$; and using $N_{i}^{[x]}$ and $N_{j}^{[x]}$ if $k_{i}=k_{j}$. The latter case relies on two properties to work correctly:

$$
\mathrm{P} 1 \text { : if } k_{i}=k_{j} \text {, then } n_{i}=n_{j} \text { and } \mathrm{P} 2:^{3} n_{i} \in V_{i}, \forall i, j \text {. }
$$

However, as discussed in Section II-C, P2 does not hold under the heterogeneous model.

To ensure $\mathrm{P} 2$, one naive solution is to first pick an arbitrary channel from $V_{i}$ as the parity channel $n_{i}$, and then rotates by the amount equal to $n_{i}$, which we call the parity-then-rotating technique. Unfortunately, this modification breaks P1. Fig. 6

\footnotetext{
${ }^{3}$ More precisely, $n_{i} \in V_{i} \backslash P_{i}$. We drop $P_{i}$ here for simplicity and will consider it later.
}

shows a counter example. The amounts of rotation in $R_{i}^{[x]}$ and $R_{j}^{[x]}$ are computed with modulo- $\left|R_{i}^{[x]}\right|$ and modulo- $\left|R_{j}^{[x]}\right|$ arithmetic since we adopt the short-cycle design for $F$ and $R$ (i.e., $k_{i}=n_{i} \bmod \left|R_{i}^{[x]}\right|$ and $k_{j}=n_{j} \bmod \left|R_{j}^{[x]}\right|$ ). Because $\left|R_{i}^{[x]}\right|=\left|R_{j}^{[x]}\right|$, it is possible that the amounts of rotation in $R_{i}$ and $R_{j}$ are the same under the modulo arithmetic (i.e., $k_{i}=n_{i} \bmod \left|R_{i}^{[x]}\right|=n_{j} \bmod \left|R_{j}^{[x]}\right|=k_{j}$ ), but $n_{i} \neq n_{j}$.

Instead of modifying the rotating-and-parity technique for P2, we argue that a key step is missing. Let $s_{t a r t}$ be the smallest element in $V_{i}$. If we can first ensure that

\section{P3: if $k_{i}=k_{j}$, then start $_{i}=$ start $_{j}$ (i.e., the capabilities} of nodes $i$ and $j$ are aligned from start),

then the problem of ensuring both P1 and P2 can be easily solved by, for example, requiring every node to pick start ${ }_{i}$ as the parity channel $n_{i}$ deterministically. ${ }^{4}$

We propose a parity-alignment technique, which establishes P3 by setting

$$
k_{i}=\left(\text { start }_{i} \bmod \left|R_{i}^{[x]}\right|\right)
$$

in $R_{i}$ in Eq. (2), and ensures $\mathrm{P} 1$ and $\mathrm{P} 2$ by letting $n_{i}=$ start $_{i}$ in $N_{i}$.

Theorem 7. Given two capability-overlapping radios $i$ and $j$ that hop to channels by following $G_{i}$ and $G_{j}$ defined by the parity-alignment technique. If these two radios have the same amount of rotation $k_{i}=k_{j}$ in $R_{i}$ and $R_{j}$, then they must rendezvous at the time-overlapping slots in $N_{i}$ and $N_{j}$.

Proof: We first prove that P3 holds due to Eq. (3). Suppose on the contrary $k_{i}=k_{j}$ but start $_{i} \neq$ start $_{j}$. Then, we have $\mid$ start $_{i}-$ start $_{j}|=c| R_{i}^{[x]}|=c| R_{j}^{[x]} \mid$ for some integer $c \geq 1$. Since $\left|R_{i}^{[x]}\right| \geq\left|V_{i}\right|$ and $\left|R_{j}^{[x]}\right| \geq\left|V_{j}\right|$, we have $\mid$ start $_{i}-$ start $_{j}|\geq| R_{i}^{[x]}|\geq| V_{i} \mid$ and $\mid$ start $_{i}-$ start $_{j}|\geq| R_{j}^{[x]}|\geq| V_{j} \mid$, implying that the radios $i$ and $j$ do not have capability-overlap, i.e., $V_{i} \cap V_{j}=\emptyset$, a contradiction.

Next, since $n_{i}=$ start $_{i}=$ start $_{j}=n_{j}$, P1 holds. Furthermore, because $n_{i}=$ start $_{i} \in V_{i}$, P2 holds as well. The radios $i$ and $j$ must rendezvous in $N_{i}$ and $N_{j}$.

In the presence of PU occupancy $P_{i}$, we can simply regard start $_{i}$ as the first available channel in $V_{i} \backslash P_{i}$ to maintain the above rendezvous guarantee.

\section{Design of Heterogeneous Hopping}

So far, we have introduced two new $\mathrm{CH}$ sequences $F$ and $G$ for radios that guarantee rendezvous in two complementary situations resulting from different capabilities of wireless devices. One remaining challenge is: how to harmonize these two sequences in one so it is runnable on a radio? Combining $F$ and $G$ is not a trivial task as we need to consider the time skews between radios and there may not exist time-overlap between slots in $F_{i}$ and $F_{j}, R_{i}$ and $R_{j}$, and $N_{i}$ and $N_{j}$ for some $i$ and $j$. The notion of cyclic quorums, as shown in Fig. 3(c), is not applicable here, as it is hard to squeeze three cyclic quorums into an $S_{i}^{[x]}$ (and $S_{j}^{[x]}$ ) for $F_{i}^{[x]}, R_{i}^{[x]}$, and $N_{i}^{[x]}$ respectively.

Instead of forcing the time-overlap between slots in $F_{i}$ and $F_{j}, R_{i}$ and $R_{j}$, and $N_{i}$ and $N_{j}$, we allow skews between

\footnotetext{
${ }^{4}$ While other rules of setting $n_{i}$ are possible, in this paper we use the simplest strategy that $n_{i}=$ start $_{i}$ to avoid distraction. In Section VII, we will motivate new rules to cope with some extreme situations.
} 


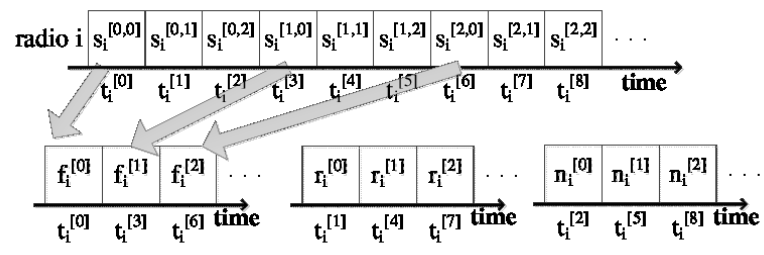

Figure 7. Two-layer design for a $\mathrm{CH}$ sequence. $S_{i}$ is divided into rounds, and each round is further divided into fixed $F_{i}$, rotating $R_{i}$, and parity $N_{i}$ subsequences.
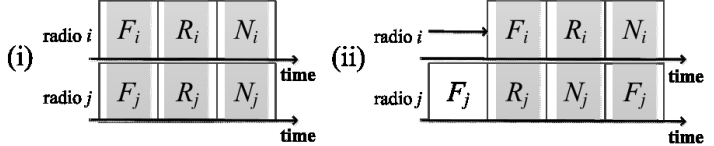

(iii)

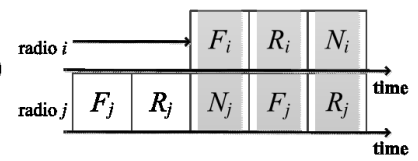

Figure 8. Time skews between two $\mathrm{CH}$ sequences of the $\mathrm{HH}$ scheme. Only two cases need to be considered: (i) $F$ with $F, R$ with $R$, and $N$ with $N$; and (ii) $F$ with $R, R$ with $N$, and $N$ with $F$.

these slots and carefully design the $\mathrm{HH}$ scheme such that it guarantees rendezvous in all possible skew cases. The $\mathrm{HH}$ scheme combines $F_{i}, R_{i}$, and $N_{i}$ into rounds (of length 3 ) to produce the $\mathrm{CH}$ sequence $S_{i}$ for each radio $i$, as:

$$
s_{i}^{[x, y]}=\left\{\begin{array}{ll}
f^{[x]}, & y=0, \\
r^{[x]}, & y=1, \\
n^{[x]}, & y=2,
\end{array} .\right.
$$

Fig. 7 shows an example. The three subsequences $F_{i}=$ $\left[f_{i}^{[0]}, f_{i}^{[1]}, \cdots\right], R_{i}=\left[r_{i}^{[0]}, r_{i}^{[1]}, \cdots\right]$, and $N_{i}=\left[n_{i}^{[0]}, n_{i}^{[1]}, \cdots\right]$ of $S_{i}$ are called fixed sequence, rotating sequence, and parity sequence, respectively. Unlike a cycle in traditional $\mathrm{CH}$ sequences, a round allows the channels from each of $F_{i}, R_{i}$, and $N_{i}$ to appear only once.

The advantage of such a two-layer design is that, first, multiple sequences can be combined without major revisions on the cycles of $F_{i}, R_{i}$, and $N_{i}$. Furthermore, in the presence of time skews between radios, the round layer with a simple structure can greatly simplify the time-overlapping cases between the slots in different subsequences, as discussed next.

\section{A. Rounding for Asynchronous Timers}

Despite of possible time skews between two radios, we only need to consider two time-overlapping cases with the aid of rounds: ${ }^{5}$ (i) $F$ with $F, R$ with $R$, and $N$ with $N$, and (ii) $F$ with $R, R$ with $N$, and $N$ with $F$, as shown in Fig. 8. Case (iii) in Fig. 8 is covered by the case (ii) as we don't distinguish $(i, j)$ from $(j, i)$.

We have discussed how to guarantee rendezvous between two radios $i$ and $j$ for case (i) in the previous section by considering the subcases (a) $\left|F_{i}^{[x]}\right| \neq\left|F_{j}^{[x]}\right|$, (b) $\left|F_{i}^{[x]}\right|=$ $\left|F_{j}^{[x]}\right| \wedge k_{i} \neq k_{j}$, and (c) $\left|F_{i}^{[x]}\right|=\left|F_{j}^{[x]}\right| \wedge k_{i}=k_{j}$.

\footnotetext{
${ }^{5}$ Recall from Fig. 2 that we can regard time skews as integer multiples of a time slot and focus on the time-overlap relationship between slots.
}

For case (ii) with subcases (a) or (c), we can use the timeoverlapping slots in $R_{i}$ and $N_{j}$ together with those in $N_{i}$ and $F_{j}$ to guarantee rendezvous. Note that $V_{i} \cap V_{j} \neq \emptyset$ implies that either start $_{i} \in V_{j}$ or start $_{j} \in V_{i}$ must be hold. If start $_{i} \in V_{j}$, then rendezvous must occur at some time-overlapping slot in $N_{i}$ and $F_{j}$ within a cycle $F_{j}^{[x]}$, as each $F_{j}^{[x]}$ will iterate over all channels in $V_{j}$. Otherwise, if start $_{j} \in V_{i}$, then rendezvous must occur at some time-overlapping slot in $R_{i}$ and $N_{j}$ within a cycle $R_{i}^{[x]}$.

For case (ii) with subcase (b), we observe that if the amount of rotation $k_{j}$ for $R_{j}$ is always greater than 0 , then we can use the time-overlapping slots between $F_{i}$ and $R_{j}$ to guarantee rendezvous thanks to Lemma 5 . As $k_{j}$ may be equal to 0 by Eq. (3), we refine the rule as

$$
k_{i}=\left(\text { start }_{i} \bmod \left|R_{i}^{[x]}\right|-1\right)+1
$$

such that $1 \leq k_{i} \leq\left|R^{[x]}\right|-1$. This creates a subtle problem: since $k_{i}$ is defined under the modulo- $\left(\left|R_{i}^{[x]}\right|-1\right)$ arithmetic now, Theorem 7 no longer holds when $\mid$ start $_{i}-$ start $_{j} \mid=$ $\left|R_{i}^{[x]}\right|-1$ and $\left|V_{i}\right|$ (or $\left|V_{j}\right|$ ) is a prime such that $\left|R_{i}^{[x]}\right|=$ $\left|V_{i}\right|$, as $\mid$ start $_{i}-\operatorname{start}_{j}|=| R_{i}^{[x]}|-1 \nsupseteq| R_{i}^{[x]}|=| V_{i} \mid$ (or $\mid$ start $_{i}-$ start $\left._{j}|=| R_{j}^{[x]}|-1 \nsupseteq| R_{j}^{[x]}|=| V_{j} \mid\right)$. To fix this, we require $\left|F_{i}^{[x]}\right|$ and $\left|R_{i}^{[x]}\right|$ to be the next prime if the capability $V_{i}$ of a radio is already a prime.

\section{B. Guarantee of Rendezvous and MTTR}

Here we show that $\mathrm{HH}$ can guarantee rendezvous and derive an upper bound for MTTR. Following the discussion thus far, without loss of generality, we consider all combinations of the cases (i) and (ii) vs. subcases (a), (b), and (c).

(i-a): we can use the time-overlapping slots in $F_{i}$ and $F_{j}$ to guarantee rendezvous, and MTTR is $O\left(\left|V_{i}\right|\left|V_{j}\right|\right)$ as stated in Theorem 6.

(i-b): we can use the time-overlapping slots in $R_{i}$ and $R_{j}$ to guarantee rendezvous, and MTTR is bounded by $O\left(\left|V_{i}\right|\left|V_{j}\right|\right)$ by Lemma 5 .

(i-c): based on Theorem 7, we can use the timeoverlapping slots in $N_{i}$ and $N_{j}$ to guarantee rendezvous. MTTR is bounded by $O\left(\left|S^{[x]}\right|\right)=O(1)$.

(ii-a): we can use the time-overlapping slots in $R_{i}$ and $N_{j}$ together with those in $N_{i}$ and $F_{j}$ to guarantee rendezvous as described earlier. MTTR is $\max \left\{O\left(\left|V_{i}\right|\right), O\left(\left|V_{j}\right|\right)\right\}$.

(ii-b): we can use the time-overlaps of $F_{i}$ and $R_{j}$ to guarantee rendezvous by regarding each $F_{i}^{[x]}$ as a rotating sequence with the amount of rotation $k_{i}=0$. Since $1 \leq k_{j} \leq\left|R_{j}\right|-1$ and $k_{i} \neq k_{j}$, the argument in case (i-b) applies here, and the MTTR is $O\left(\left|V_{i}\right|\left|V_{j}\right|\right)$.

(ii-c): the argument in case (ii-a) applies here.

In summary, the $\mathrm{HH}$ scheme can guarantee rendezvous in asynchronous CRNs consisting of heterogeneous wireless devices, and MTTR is bounded by $O\left(\left|V_{i}\right|\left|V_{j}\right|\right)$.

\section{Performance Evaluation}

We now evaluate the performance of $\mathrm{HH}$. Since it is, in practice, difficult to synchronize timers, we focus on asynchronous environments. 


\section{A. Simulation Setup}

$\mathrm{HH}$ is implemented at MAC layer using the Network Simulator 3 (NS3). We modify WiFi MAC to support the channel hopping function, and adopt IEEE 802.11b as the MAC-layer protocol. The duration of a time slot is set to $100 \mathrm{~ms}$. Each radio is capable of switching among channels at consecutive slots, and receives or transmits one packet at a time. Also, radios can detect whether a channel is idle or not. We set $|U|=600$ by default, which is shown in [15] to be realistic in a large-scale CRN consisting of heterogeneous radios. We generate $t$ types of heterogeneous radios, and there are $r$ homogeneous radios of each type. By default, we set $t=5$ and $r=3$. The capability size of radios in each type ranges from 12 to 50 . We control the degree of capability-overlap between radio types by varying the overlapping ratio - the fraction of the number of channels in $U$ that can be sensed by more than two radios. By default, the overlapping ratio is set to 0.1 . The ratio, called the $P U$-occupied ratio, of the number of PUoccupied channels to $|U|$ is set to 0.1 . PU-occupied channels are randomly selected from $U$. To focus on the impact of heterogeneous radios at MAC layer, we place the radios such that their ranges cover each other. In effect, we simulate a group of neighboring radios in a CRN, so our measurements are not influenced by the protocols in network and application layers. Each simulation run lasts for 600 seconds, and each data point is obtained by averaging 120 runs.

We compare $\mathrm{HH}$ with proposals [23], [25] (denoted as BASELINE) that employ the ideas similar to the short-cycle technique where each radio hops to channels in its capability set one-by-one. However, they do not guarantee rendezvous of all pairs under the heterogeneous model. We also compare $\mathrm{HH}$ with Modified Modular Clock [25] (denoted as RAND), a state-of-the-art randomized scheme. MMC guides radios to rendezvous with a high probability, in expected TTR of $O\left(\left|V_{i}\right|\left|V_{j}\right|\right)$ slots, but it cannot guarantee rendezvous either. We also extend Jump-Stay (JS) [20], a CH scheme that guarantees rendezvous and has superior performance under the asymmetric model, for the heterogeneous model by creating a nominal capability $V=U$ as described in Section II-C. Note that in JS, every channel in $V_{i} \backslash P_{i}$ takes turn to be the parity channel in successive cycles. Therefore, this extended version of JS can avoid the problem shown in Fig. 4(b) and guarantee rendezvous, at the cost of high $O\left(|U|^{2}\right)$ MTTR.

\section{B. Verifying the Guarantee of Rendezvous}

In this series of simulations, we verify the guarantee of rendezvous given by $\mathrm{HH}$ under the heterogeneous model. We study the success rate, the ratio of the number of $\mathrm{CR}$ pairs which rendezvous before the end of a simulation run to all pairs, achieved by each $\mathrm{CH}$ scheme while varying the overlapping ratio, PU-occupied ratio, and capability size respectively. As we can see in Figs. 9(a)-(c), both the HH and extended JS achieve a $100 \%$ success rate under various conditions, whereas RAND and BASELINE cannot due to their lack of rendezvous guarantee. In particular, the BASELINE gives lower than $80 \%$ success rate in most cases.

We observe that, although without any rendezvous guarantee, RAND achieves a very high success rate (above $98 \%$ in all cases). This is because RAND yields the expected success rate equal to 1 in long term. So, the success rate goes up higher as the simulation time gets longer. However, there is no guarantee on when will RAND give a $100 \%$ success rate (or above a

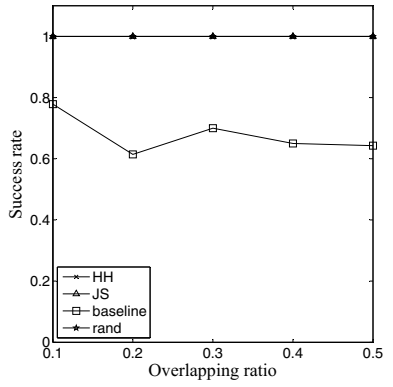

(a)

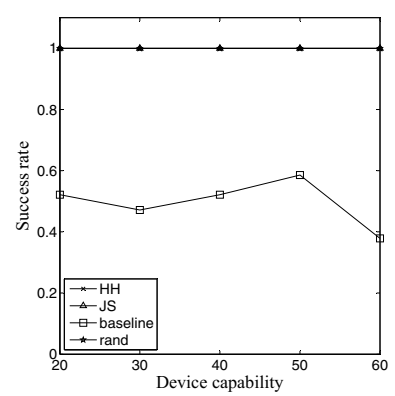

(c)

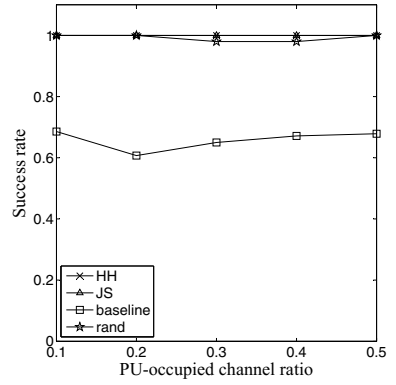

(c)

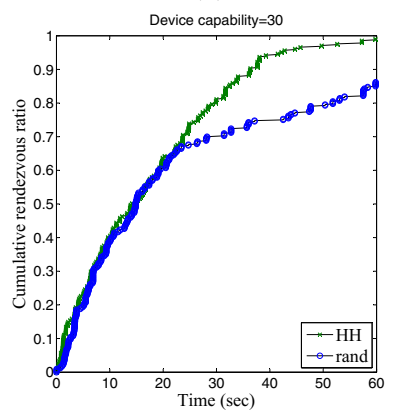

(d)
Figure 9. (a)-(c) Success rate under varying conditions. (d) Indefinite cumulative rendezvous ratio given by RAND.

certain threshold). To see this, we fix the capability size $V_{i}$ of all radios to 25 , let $\left|V_{i} \cap V_{j}\right|=1$, and show how the success rate changes with the simulation time in Fig. 9(d). Benefiting from $O\left(\left|V_{i}\right|\left|V_{j}\right|\right)$ MTTR, HH can guarantee a $100 \%$ success rate within $25^{2}$ slots (roughly 63 seconds given $100 \mathrm{~ms}$ slot duration). On the other hand, RAND yields only an $85 \%$ success rate within this time bound. The indefinite success rate prevents RAND from supporting QoS at the network or application layer.

\section{Time To Rendezvous}

Next, we study the average TTR of CR pairs given by different $\mathrm{CH}$ schemes. ${ }^{6}$ Note that BASELINE and RAND perform very similarly in terms of average TTR, so we plot BASELINE only. Figs. 10(a)-(c) show the average TTR under various conditions. Intuitively, when the overlapping ratio gets lower (or the PU-occupied ratio gets higher), the average TTR will raise because there are less common channels between CR pairs. Nevertheless, as shown in Figs. 10(a) and (b), HH and BASELINE are affected less by the changes of these two ratios thanks to the short-cycle design. The short-cycle design, however, makes $\mathrm{HH}$ and BASELINE more sensitive to the capability sizes of radios, as shown in Figs. 10(c).

Overall, $\mathrm{HH}$ is able to yield the average TTR as low as the BASELINE and RAND do, despite the fact that they cannot guarantee rendezvous. When compared to the extended JS which guarantees rendezvous, $\mathrm{HH}$ is $5 \times$ to $10 \times$ faster. To see how $\mathrm{HH}$ outperforms the extended JS, we evaluate the success rate over simulation time. With default settings, Fig. 10(d) shows that most CR pairs adopting the extended JS scheme rendezvous after 7 seconds, while $\mathrm{HH}$ guides almost all radios

\footnotetext{
${ }^{6}$ We do not count the TTR of a radio pair in the average TTR if they fail to rendezvous before the end of a simulation run (600 seconds).
} 


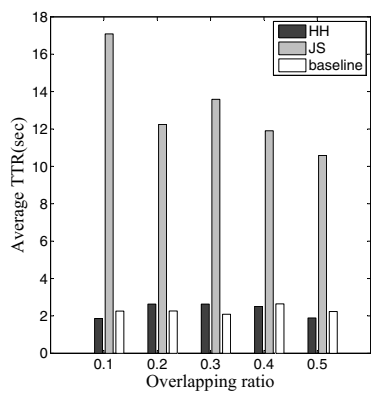

(a)

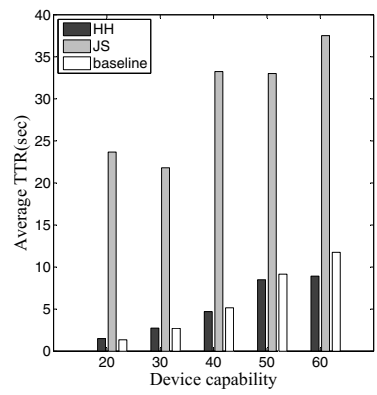

(c)

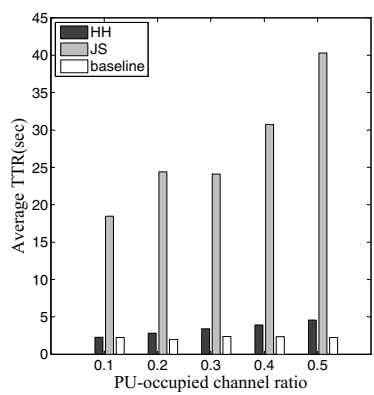

(b)

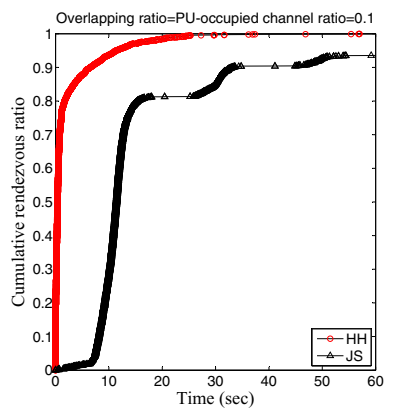

(d)
Figure 10. (a)-(c) Average TTR under varying conditions. (d) Slow-growing cumulative rendezvous ratio yielded by extended JS.

to rendezvous within 25 seconds. This is due mainly to the long cycle length $|U|$ adopted by the extended JS and coincides with the $O\left(|U|^{2}\right)$ MTTR of extended JS versus $O\left(\left|V_{i}\right| V_{j}||\right)$ MTTR of HH.

\section{Degree of Congestion}

One merit of channel hopping, as compared to the Common Control Channel (CCC) approach, is to guide different CR pairs to rendezvous in different channels, thereby avoiding congestion. To study the degree of congestion incurred by $\mathrm{HH}$, we define the load of a channel $c$ as the ratio of the maximum number of radios that hop to $c$ at the same time, to the total number of radios $|D|$. We use the maximum channel load of all channels in $U$ to measure the degree of congestion, and study this metric under different degrees of heterogeneity. We control the degree of heterogeneity by setting the total number of radios to 20 , and varying the number $t$ of heterogeneous radio types from 1 to 20 . There are $r=20 / t$ homogeneous radios of each type. A larger (resp. lower) $t$ implies higher heterogeneity (resp. homogeneity).

Fig. 11 shows the maximum channel load yielded by the $\mathrm{HH}$, extended JS, and CCC during the simulation runs. In CCC, we reserve a channel available to all radios and use this channel as the common control channel. So, its maximum channel load is always 1 . HH is shown to provide a much lower channel load than CCC and is effective in avoiding congestion as most existing $\mathrm{CH}$ schemes. Note that in the presence of high homogeneity $(t \leq 2)$, $\mathrm{HH}$ incurs $10 \%$ to $15 \%$ higher channel load than the extended JS. However, high homogeneity is not likely in large-scale CRNs consisting of stranger CRs. In the presence of mid-to-high heterogeneity $(t \geq 3)$, the channel loads yielded by $\mathrm{HH}$ are comparable (less than $5 \%$ difference) to those given by the extended JS.

\section{RELATED WORK}

Traditionally, $\mathrm{CH}$ schemes under the asymmetric model have been designed either for synchronous or asynchronous environments. In synchronous environments, the timers of all radios are is synchronized, so that they can start and hop to a channel simultaneously [3], [17], [24], [26]. However, timer synchronization may be difficult, or very expensive, in practice. The asynchronous $\mathrm{CH}$ schemes have thus been proposed. Bian et al. [4], [5], [7] proposed A-MOCH and Sym-ACH. A-MOCH requires senders and receivers to be known in advance, which is unlikely before rendezvous. Sym$\mathrm{ACH}$ assumes each node to have a unique ID. The MAC (usually 48 bits) address seems to be the only choice, causing $2^{48} \mathrm{O}\left(|V|^{2}\right)$ MTTR. DaSilva et al. [14] proposed another $\mathrm{CH}$ scheme under the condition that all radios have the same available channels. Zhang et al. [27] proposed Asyn-ETCH that requires the schedules with rendezvous guarantees to be constructed in advance, but it is unlikely for radio $i$ to preconstruct the overlapping schedules for all unknown $V_{j}$. Lin et al. [20] proposed the JS that uses the jump and stay patterns of lengths $2|V|$ and $|V|$, respectively, and is the first approach that guarantees rendezvous in general asynchronous environments. However, all of the above approaches assume the asymmetric model.

The short-cycle technique is not new and is used by Theis et al. [25]. However, this technique was introduced as an extension of the prime-and-rotating where there are still a rotation amount from cycle to cycle controlled by the "rate" parameter and a periodically-size-changing augmented capability, and therefore cannot guarantee rendezvous if two radios continually make the same decision (either on the rate parameter or on the size of augmented capability) to construct their $\mathrm{CH}$ sequences.

Romaszko et al. proposed the MtQS-DSrdv [23] that verifies rendezvous under the heterogeneous model when $\left|V_{i}\right|,\left|V_{j}\right| \leq$ 8 using simulation, but does not provide any theoretical guarantee for other cases. In fact, we can give a counter example that the scheme cannot guarantee rendezvous in a general case $V_{i} \neq V_{j}$. We briefly describe the MtQS-DSrdv as follows: each radios $i$ (i) maps slots to a grid of size $\left|V_{i}\right| \cdot\left(2\left|V_{i}\right|-1\right)$, where each row $r$ has slots from $t_{i}^{\left[0+r\left(2\left|V_{i}\right|-1\right)\right]}$ to $t_{i}^{\left[2\left|V_{i}\right|-2+r\left(2\left|V_{i}\right|-1\right)\right]}$; (ii) assigns each channel in $V_{i}$ to a randomly selected column from the first $\left|V_{i}\right|$ columns; and (iii) assigns each channel in $V_{i}$ to a randomly selected row of the rest empty slots. An example is given in the Fig. 12(a). While $V_{i} \neq V_{j}$, there may not be rendezvous, as shown in Fig. 12(b). Currently, there is no known solution that can guarantee rendezvous under the heterogeneous model.

\section{CONCLUSION}

We proposed the HH scheme, which employs a twolayer design and harmonizes the fixed-short-cycle and parityalignment techniques to guide radios to rendezvous in two complementary situations resulting from different capabilities 


\begin{tabular}{l|l|l|l|l|l|l|}
\hline 2 & 0 & 1 & 3 & 2 & 2 & 2 \\
\hline 2 & 0 & 1 & 3 & 1 & 1 & 1 \\
\hline 2 & 0 & 1 & 3 & 3 & 3 & 3 \\
\hline 2 & 0 & 1 & 3 & 0 & 0 & 0 \\
\hline
\end{tabular}

(a)

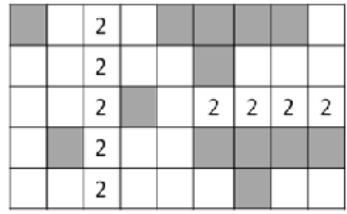

(b)
Figure 12. (a) The grid for $V_{i}=\{0,1,2,3\}$ (b) Let $\left|V_{j}\right|=5$ and $V_{i} \cap V_{j}=$ $\{2\}$, the grid of $V_{j}$ (where only the common channel 2 is shown) cannot guide $V_{j}$ to rendezvous with $V_{i}$ in a cycle of $O\left(\left|V_{j}\right|^{2}\right)$ slots (neither $O\left(\left|V_{i}\right|\left|V_{j}\right|\right)$ ) slots). The shaded slots mark where $V_{i}$ hops to the channel 2 under some particular time skew.

of wireless devices. The rendezvous of all pairs is guaranteed without the need of timer synchronization between radios. Our extensive simulation results have shown that the proposed schemes achieve close to a $90 \%$ reduction in average TTR without incurring significant overhead in congestion as compared to the naive extensions of previous solutions.

As shown in Section V, HH may yield higher channel load in an extreme situation where most radios in a network have the same capability. This is because we use the simplest strategy $n_{i}=$ start $_{i}$ to set the parity channel (to ensure P1 and P2 after establishing P3 using Eq. (3), as described in Section III-B), and thus those neighboring radios sharing the same rotation amount $k_{i}$ in the rotating subsequence $R_{i}$ will all rendezvous using the same parity channel, $s_{a r t}$, in $N_{i}$. One way to overcome this is to let $n_{i}$ iterate through all channels in $V_{i} \backslash P_{i}$ in successive cycles $N_{i}^{[0]}, N_{i}^{[1]}, \cdots$, as used by JS. However, this strategy requires new techniques to deal with the time-overlapping cases (ii-a) and (ii-c) in Section IV. This is a matter of our future inquiry.

\section{REFERENCES}

[1] I.F. Akyildiz, W.Y. Lee, M.C. Vuran, and S. Mohanty. Next generation/dynamic spectrum access/cognitive radio wireless networks: a survey. Computer Networks, 50(13):2127-2159, 2006.

[2] R. Bagheri, A. Mirzaei, S. Chehrazi, M. Heidari, M. Lee, M. Mikhemar, M. Tang, and A. Abidi. An $800 \mathrm{mhz}$ to $5 \mathrm{ghz}$ software-defined radio receiver in $90 \mathrm{~nm}$ cmos. In IEEE Int'l Solid-State Circuits Conference (ISSCC), pages 1932-1941, 2006.

[3] P. Bahl, R. Chandra, and J. Dunagan. Ssch: slotted seeded channel hopping for capacity improvement in ieee 802.11 ad-hoc wireless networks. In Proc. of ACM Int'l Conf. on Mobile computing and networking (MobiCom), pages 216-230, 2004.

[4] K. Bian and J. Park. Maximizing rendezvous diversity in rendezvous protocols for decentralized cognitive radio networks. IEEE Transactions on Mobile Computing, 2012.

[5] K. Bian and J.M. Park. Asynchronous channel hopping for establishing rendezvous in cognitive radio networks. In Proc. of IEEE Int'l Conf. on Computer Communications (INFOCOM), pages 236-240, 2011.

[6] K. Bian, J.M. Park, and R. Chen. A quorum-based framework for establishing control channels in dynamic spectrum access networks. In Proc. of ACM Int'l Conf. on Mobile computing and networking (MobiCom), pages 25-36, 2009.

[7] K. Bian, J.M. Park, and R. Chen. Control channel establishment in cognitive radio networks using channel hopping. IEEE Journal on Selected Areas in Communications, 29(4):689-703, 2011.

[8] V. Brik, E. Rozner, S. Banerjee, and P. Bahl. Dsap: a protocol for coordinated spectrum access. In IEEE Int'l Symp. on New Frontiers in Dynamic Spectrum Access Networks (DySPAN), pages 611-614, 2005.

[9] M.M. Buddhikot, P. Kolodzy, S. Miller, K. Ryan, and J. Evans. Dimsumnet: new directions in wireless networking using coordinated dynamic spectrum. In IEEE Int'l Symp. on World of Wireless Mobile and Multimedia Networks (WoWMoM), pages 78-85. Ieee, 2005.
[10] D. Cabric, S.M. Mishra, and R.W. Brodersen. Implementation issues in spectrum sensing for cognitive radios. In Proc. of Asilomar Conf. on Signals Systems and Computers, pages 772-776, 2004.

[11] Eugene Chai, Jeongkeun Lee, Sung-Ju Lee, Raul Etkin, and Kang G Shin. Building efficient spectrum-agile devices for dummies. In Proc. of ACM Int'l Conf. on Mobile computing and networking (MobiCom), pages 149-160, 2012

[12] C. Cordeiro, K. Challapali, D. Birru, and N. Sai Shankar. Ieee 802.22: the first worldwide wireless standard based on cognitive radios. In IEEE Int'l Symp. on New Frontiers in Dynamic Spectrum Access Networks (DySPAN), pages 328-337, 2005.

[13] C. Cormio and K.R. Chowdhury. Common control channel design for cognitive radio wireless ad hoc networks using adaptive frequency hopping. Ad Hoc Networks, 8(4):430-438, 2010.

[14] L.A. DaSilva and I. Guerreiro. Sequence-based rendezvous for dynamic spectrum access. In IEEE Int'l Symp. on New Frontiers in Dynamic Spectrum Access Networks (DySPAN), pages 1-7, 2008.

[15] A. Ghasemi and E.S. Sousa. Spectrum sensing in cognitive radio networks: requirements, challenges and design trade-offs. IEEE Communications Magazine, 46(4):32-39, 2008.

[16] M. Ingels, V. Giannini, J. Borremans, G. Mandal, B. Debaillie, P. Van Wesemael, T. Sano, T. Yamamoto, D. Hauspie, J. Van Driessche, et al. A $5 \mathrm{~mm}^{2} 40 \mathrm{~nm}$ lp cmos transceiver for a software-defined radio platform. IEEE Journal of Solid-State Circuits, 45(12):2794-2806, 2010.

[17] Y.R. Kondareddy and P. Agrawal. Synchronized mac protocol for multi-hop cognitive radio networks. In Proc. of IEEE Int'l Conf. on Communications (ICC), pages 3198-3202, 2008.

[18] S. Krishnamurthy, M. Thoppian, S. Venkatesan, and R. Prakash. Control channel based mac-layer configuration, routing and situation awareness for cognitive radio networks. In IEEE Military Communications Conference (MILCOM), pages 455-460, 2005.

[19] L. Le and E. Hossain. A mac protocol for opportunistic spectrum access in cognitive radio networks. In IEEE Wireless Communications and Networking Conference (WCNC), pages 1426-1430, 2008.

[20] Z. Lin, H. Liu, X. Chu, and Y.W. Leung. Jump-stay based channelhopping algorithm with guaranteed rendezvous for cognitive radio networks. In Proc. of IEEE Int'l Conf. on Computer Communications (INFOCOM), pages 2444-2452, 2011.

[21] W.S. Luk and T.T. Wong. Two new quorum based algorithms for distributed mutual exclusion. In Proc. of IEEE Int'l Conf. on Distributed Computing Systems (ICDCS), pages 100-106, 1997.

[22] J. Pérez-Romero, O. Salient, R. Agustí, and L. Giupponi. A novel ondemand cognitive pilot channel enabling dynamic spectrum allocation. In IEEE Int'l Symp. on New Frontiers in Dynamic Spectrum Access Networks (DySPAN), pages 46-54, 2007.

[23] S. Romaszko and P. Mahonen. A rendezvous protocol with the heterogeneous spectrum availability analysis for cognitive radio ad hoc networks. Journal of Electrical and Computer Engineering, 2012.

[24] C.F. Shih, T.Y. Wu, and W. Liao. Dh-mac: A dynamic channel hopping mac protocol for cognitive radio networks. In Proc. of IEEE Int'l Conf. on Communications (ICC), pages 1-5, 2010.

[25] N.C. Theis, R.W. Thomas, and L.A. DaSilva. Rendezvous for cognitive radios. IEEE Transactions on Mobile Computing, 10(2):216-227, 2011.

[26] C. Xin, M. Song, L. Ma, and C.C. Shen. An approximately optimal rendezvous scheme for dynamic spectrum access networks. In Proc. of IEEE Int'l Conf. on Gloal Telecommunications (GLOBECOM), pages $1-5,2011$.

[27] Y. Zhang, Q. Li, G. Yu, and B. Wang. Etch: Efficient channel hopping for communication rendezvous in dynamic spectrum access networks. In Proc. of IEEE Int'l Conf. on Computer Communications (INFOCOM), pages 2471-2479, 2011. 